\title{
INTERLEAVED ORTHOGONAL FREQUENCY DIVISION MULTIPLEXING SYSTEM
}

\author{
V.G.S.Prasad and K.V.S.Hari \\ Department of Electrical CommunicationEngineering, \\ Indian Institute of Science, Bangalore 560 012, India \\ vprasad@protocol.ece.iisc.ernet.in, hari@ece.iisc.ernet.in
}

\begin{abstract}
A new orthogonalfrequency division multiplexing(OFDM) system for wireless communications over frequency selective fading channels is presented. The proposed interleaved orthogonal frequency division multiplexing (IOFDM) system promises higher code rate as compared to the conventional OFDM system without bandwidth expansion and with very slight increase in computational complexity. Simulation results verify that the performance, in terms of bit error rate, of the proposed IOFDM system is very close to the conventional OFDM system.
\end{abstract}

\section{INTRODUCTION}

In recent years, orthogonal frequency division multiplexing (OFDM) has been adopted as a standard for applications like digital audio broadcasting (DAB), digital video broadcasting (DVB) and wireless LANs etc. In order to avoid inter block interference (IBI) between OFDM blocks arising due to the frequency selective nature of the channel, either cyclic prefix $(\mathrm{CP})$ or zero padding $(\mathrm{ZP})$ is added to each OFDM block before transmission [1], [2]. The length of the CP/ZP should be longer than the channel delay profile to avoid IBI. Due to this CP/ZP, the code rate of the communication system reduces. In this paper, we propose a new scheme called the interleaved OFDM (IOFDM) system, which enhances the code rate compared to the conventional OF'DM system.

\section{ORTHOGONAL FREQUENCY DIVISION MULTIPLEXING SYSTEM}

In an OFDM system [3], [4], the bit stream is mapped to an information symbol sequence $s(n)$ using a modulation scheme. The sequence $s(n)$ is divided into blocks, ${ }^{1} \underline{s}(n)=$ $[s(n K), \ldots, s(n K+K-1)]^{T}$, of length $\mathrm{K}$. Then $\underline{s}(n)$ is further precoded into another block $\underline{u}(n)$, of length $K$, through a precoder matrix $C$ such that$$
\underline{\underline{u}}(n)=[u(n K), \ldots, u(n K+K-1)]^{T}=C \underline{s}(n) .
$$

${ }^{1} T$ stands for transpose. Underline denotes vectors.
}

The role of $C$ is to effectively convert a frequency selective fading channel into a number of flat fading channels.

Let us assume that the frequency selective channel is FIR in nature and the upper bound on its order is assumed to be known i.e., $h(l)=0, \forall l \notin[0, L]$. Let us construct $\underline{\underline{x}}^{\prime}(n)$, of length $N=K+L$, as follows

$$
\begin{aligned}
\underline{x}^{\prime}(n) & =[u(n K), \ldots, u(n K+K-1), 0, \ldots, 0]^{T} \\
& =\left[x^{\prime}(n N), \ldots, x^{\prime}(n N+N-1)\right]^{T} .
\end{aligned}
$$

The last $L$ elements of the block $\underline{x}^{\prime}(n)$ are set to zero. This process is known as zero padding. $K$ and $N$ are called the OFDM block length and the transmitted OFDM block length, respectively. The sequence $x^{\prime}(n)$ is then serially transmitted through a transmitting antenna. Because of ZP, the channel induced IBI between the blocks of $\underline{x}^{\prime}(n)$ is avoided and one can focus at each received block separately. At the receiver, the received OFDM block, in the absence of noise, is given by

$$
\underline{y}^{\prime}(n)=H \underline{x}^{\prime}(n),
$$

where $H$ is the channel convolution matrix of size $N \times N$ with the $(k, l)$ th element being $h(k-1)$.

Define the followingz-transforms.

$C_{k}(z)=\sum_{\mathrm{h}=\mathrm{d}}^{\mathrm{n}} c_{k}(m) z^{-m}, H(z)=\sum_{\mathrm{f}_{n}=0}^{L} h\left(m, z^{-m}\right.$ and $Y^{\prime}(n ; z)=\sum_{m=0}^{N-1} y^{\prime}(n N+m) z^{-m}$. Then, the $z$. transform of (3) is given by

$$
\begin{aligned}
Y^{\prime}(n ; z) & =H(z) X^{\prime}(n ; z) \\
& =H(z) \sum_{k=0}^{K-1} C_{k}(z) s(n K+k) .
\end{aligned}
$$

Let us choose $K$ distinct points $\left\{\rho_{l}\right\}_{l=0}^{K-1}$ on the complex z-plane. The matrix $C$ is designed such that

$$
C_{k}\left(\rho_{l}\right)=a \delta(k-4, \quad \forall k, l \in[0, \mathrm{~K}-1],
$$

${ }^{2} c_{k}(m)$ denotes the element present in $(m+1) s t$ row and $(k+1) s t$ column of C. $C_{k}(z)$ denotes the $z$-transform of $(k+1)$ st column of C. 
where $a$ is chosen to impose transmission power constraint ${ }^{3}$ i.e., $E\left(\underline{s}(n)^{\mathcal{H}} \underline{s}(n)\right)=E\left(\underline{u}(n)^{\mathcal{H}} \underline{u}(n)\right) \leq P_{\text {tot }}$. (5) enables proper data recovery at the receiver. $C$ can be constructed by considering $C_{k}(z)$ to be an FIR filter of order $K-\mathbf{1}$ with zeros being $\left\{\rho_{l}\right\}_{l=0, l \neq k}^{K-1}[2]$. For $\rho_{l}, \forall l \in[0, K-1]$,

$\left.Y^{\prime}(n ; z)\right|_{z=\rho_{l}} \stackrel{\mathrm{A}}{=} Y^{\prime}\left(n ; \rho_{l}\right)=H\left(\rho_{l}\right) \sum_{k=0}^{\mathrm{K}-\mathbf{1}} C_{k}\left(\rho_{l}\right) s(n K+k)$.

Using (5), (6) is simplified as

$$
Y^{\prime}\left(n ; \rho_{l}\right)=a H\left(\rho_{l}\right) s(n K+l), \quad \forall l \in[0, K-1] .
$$

Using (7), $s(n)$ can be estimated given the knowledge of the channel state information (CSI). The code rate of the OFDM system, denoted by $\eta$, is $\eta=K / N=K /(K+$ $L)$. A new scheme is presented in the next section which inproves the code rate.

\section{INTERLEAVED ORTHOGONAL FREQUENCY DIVISION MULTIPLEXING SYSTEM}

In this section, the transmitter and receiver sections of the proposed IOFDM system are presented.

\subsection{IOFDM Transmitter}

The block diagram in Fig. 1 describes the baseband model of an IOFDM transmitter section. Let us consider two consecutive symbol blocks $\underline{s}(2 n)$ and $\underline{s}(2 n+1)$. The corresponding precoded blocks are $\underline{u}(2 n)$ and $\underline{u}(2 n+1)$.

Idea: Construct $\underline{x}(n)$, of length $M=2 K+L$, by interleaving the elements of $\underline{u}(2 n)$ and $\underline{u}(2 n+1)$ and padding $L$ zeros as follows

$$
\begin{aligned}
\underline{x}(n)= & {[u(2 n K), u((2 n+1) K), u(2 n K+1), \ldots,} \\
& u(2 n K+K-1), u((2 n+l) K+K-1), \\
& 0, \ldots, 0]^{T} \\
= & {[x(n M), \ldots, x(n M+M-1)]^{T} . }
\end{aligned}
$$

Because of interleaving, we call it as IOFDM system. The IOFDM block length is $2 K$ and the transmitted IOFDM block length is $M$.

\subsection{IOFDM Receiver}

The block diagram in Fig. 2 describes the baseband model of an IOFDM receiver section. Let us assume that the channel is constant over a transmitted IOFDM block. Similar to

\footnotetext{
${ }^{3} E()$ and $\mathcal{H}$ stand for expectation and complex conjugate transpose, respectively. $P_{t o t}$ denotes the total power allowed.
}

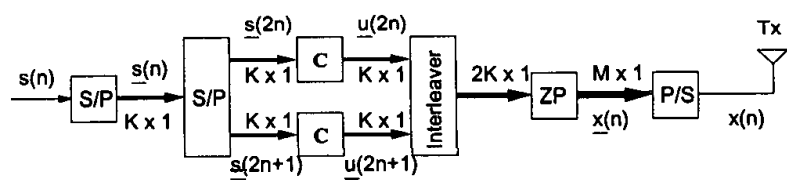

S/P : Serial to Parallel conversion. P/S : Parallel to Serial conversion. ZP :Zero Padding.

Fig, 1. Transmitter section of IOFDM system

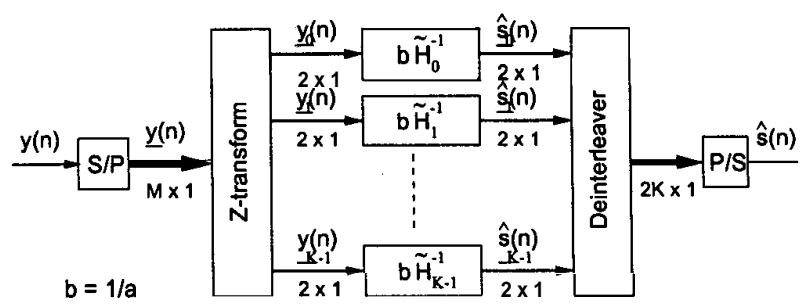

Fig. 2. Receiver section of IOFDM system

(3), the received IOFDM block, in the absence of noise, is given by

$$
y(n)=H \underline{x}(n) .
$$

The z-transform of (9) is given by

$$
\begin{aligned}
Y(n ; z)= & H(z) \sum_{\mathbf{m}=\mathbf{0}}^{\mathbf{M}-\mathbf{1}} x(n M+m) z^{-m} \\
= & H(z)\left(\sum_{\mathbf{m}=\mathbf{0}}^{K-1} u(2 n K+m) z^{-2 m}+\right. \\
& \left.z^{-1} \sum_{\mathbf{m}=\mathbf{0}}^{\mathbf{K}-\mathbf{1}} u((2 n+l) K+m) z^{-2 m}\right) \\
= & H(z)\left(\sum_{k=0}^{K-1} C_{k}\left(z^{2}\right) s(2 n K+k)+\right. \\
& \left.z^{-1} \sum_{k=0}^{\mathbf{K}-1} C_{k}\left(z^{2}\right) s((2 n+1) \mathrm{K}+k)\right) .
\end{aligned}
$$

Let us choose $2 K$ distinct points $\left\{r_{m}\right\}_{m=0}^{2 K-1}$ on the complex z-plane such that

$$
\left\{r_{m}\right\}_{m=0}^{K-1}=\left\{\rho_{l}^{1 / 2}\right\}_{l=0}^{K-1},\left\{r_{m}\right\}_{m=K}^{2 K-1}=\left\{-\rho_{l}^{1 / 2}\right\}_{l=0}^{K-1} .
$$

For $z=r_{l}, \forall l \in[0, K-1]$,

$$
\begin{aligned}
Y\left(n ; r_{l}\right)= & H\left(r_{l}\right)\left(\sum_{k=0}^{K-1} C_{k}\left(r_{l}^{2}\right) s(2 n K+k)+\right. \\
& \left.r_{l}^{-1} \sum_{k=0}^{K-1} C_{k}\left(r_{l}^{2}\right) s((2 n+1) K+k)\right) .
\end{aligned}
$$


Using (5) and (11), (12) can be simplified as follows

$$
\begin{aligned}
Y\left(n ; \rho_{l}^{1 / 2}\right)= & a H\left(\rho_{l}^{1 / 2}\right)(s(2 n K+1)+ \\
& \left.\rho_{l}^{-1 / 2} s((2 n+1) \mathrm{K}+1)\right) .
\end{aligned}
$$

Similarly, for $z=r_{K+l}, \forall l \in[0, K-1]$, we can obtain

$$
\begin{aligned}
Y\left(n ;-\rho_{l}^{1 / 2}\right)= & a H\left(-\rho_{l}^{1 / 2}\right)(s(2 n K+l)- \\
& \left.\rho_{l}^{-1 / 2} s((2 n+\mathbf{l}) \mathbf{K}+1)\right) .
\end{aligned}
$$

In order to decode $s(n)$, we define $y_{-}(n)=\left[Y\left(n ; \rho_{l}^{1 / 2}\right)\right.$, $\left.Y\left(n ;-\rho_{l}^{1 / 2}\right)\right]^{T}$ and rewrite (13) and (14) for a given $l$ as

$$
\underline{y}_{l}(n)=a \tilde{H}_{l} \underline{s}_{l}(n), \quad \forall l \in[0, K-1],
$$

where

$$
\tilde{H}_{l}=\left[\begin{array}{cc}
H\left(\rho_{l}^{1 / 2}\right) & \rho_{l}^{-1 / 2} H\left(\rho_{l}^{1 / 2}\right) \\
H\left(-\rho_{l}^{1 / 2}\right) & -\rho_{l}^{-1 / 2} H\left(-\rho_{l}^{1 / 2}\right)
\end{array}\right],
$$

$\underline{s}_{l}(n)=[s(2 n K+l), s((2 n+1) K+l)]^{T}$. It is easy to see that $\tilde{H}_{l}$ is full rank if $H\left(\rho_{l}^{1 / 2}\right) \neq 0$ and $H\left(-\rho_{l}^{1 / 2}\right) \neq 0$. The estimate of $\underline{s}_{l}(n)$ can be obtained as

$$
\hat{\underline{s}}_{l}(n)=\frac{1}{a} \tilde{H}_{l}^{-1} \underline{y}_{l}(n) .
$$

Remarks:

- Compared to the conventionalOFDM system, which considers $\mathrm{K}$ distinct points $\left\{\rho_{l}\right\}_{l=0}^{K-1}$ on the complex $\mathrm{z}$-plane, the IOFDM system considers $2 \mathrm{~K}$ distinct points $\left\{r_{m}\right\}_{m=0}^{2 K-1}$ on the complex z-plane. This implies that we need additional constraints on channel's impulse response $H(z)$.

- In presence of noise i.e., $\underline{y}_{l}(n)=a \tilde{H}_{l} \underline{\underline{s}}_{l}(n)+\underline{w}_{l}(n)$, where $\underline{w}_{l}(n)$ denotes the appropriate noise vector, the estimate $\underline{\hat{s}}_{l}(n)=\frac{1}{a} \tilde{H}_{l}^{-1} \underline{\tilde{y}}_{l}(n)$.

- The code rate of the IOFDM system, denoted by $\eta_{I}$, is $\eta_{I}=2 K / M=2 K /(2 K+L)$. The ratio, $\eta_{I} / \eta$, is greater than one, which is very desirable. It is also

\begin{tabular}{|c|c|c|c|}
\hline Twelve 1 & h TU Model & \multicolumn{2}{|c|}{ Twelve path HT Model } \\
\hline $\begin{array}{c}\text { Relative } \\
\text { Time }(\mu s)\end{array}$ & $\begin{array}{l}\text { Avg. Relative } \\
\text { Power(\&) }\end{array}$ & $\begin{array}{c}\text { Relative } \\
\text { Time }(\mu s)\end{array}$ & $\begin{array}{l}\text { Avg. Relative } \\
\text { Power(\&) }\end{array}$ \\
\hline 0.0 & -4.0 & 0.0 & -10.0 \\
\hline 0.2 & -3.0 & 0.2 & -8.0 \\
\hline 0.4 & 0.0 & 0.4 & -6.0 \\
\hline 0.6 & -2.0 & 0.6 & -4.0 \\
\hline 0.8 & -3.0 & 0.8 & 0.0 \\
\hline 1.2 & -5.0 & 2.0 & 0.0 \\
\hline 1.4 & -7.0 & 2.4 & -4.0 \\
\hline 1.8 & -5.0 & 15.0 & -8.0 \\
\hline 2.4 & -6.0 & 15.2 & -9.0 \\
\hline 3.0 & -9.0 & 15.8 & -10.0 \\
\hline 3.2 & -11.0 & 17.2 & -12.0 \\
\hline 5.0 & -10.0 & 20.0 & -14.0 \\
\hline
\end{tabular}
observed that the redundancy due to $\mathrm{ZP}$ is less by a factor of 2 .

- The IOFDM system requires additional computation in terms of a ' 2 × 2 matrix inversion operation' for every two symbols. able 1. GSM Channel Models

\subsection{An example of an IOFDM system}

Let us choose $K$ distinct points $\left\{\rho_{l}\right\}_{l=0}^{K-1}=\left\{e^{j \frac{2 \pi l}{K}}\right\}_{l=0}^{K-1}$. For this choice of $\left\{\rho_{l}\right\}_{l=0}^{K-1}$, the precoder $C$ becomes an IDFT matrix with the element $c_{k}(m)=\frac{1}{\sqrt{K}} e^{j \frac{2 \pi m k}{K}}$ and $a=\sqrt{K}$. Using (11), the $2 \mathrm{~K}$ distinctpoints $\left\{r_{m}\right\}_{m=0}^{2 K-1}$ are as follows

$$
\left\{r_{m}\right\}_{m=0}^{K-1}=\left\{e^{j \frac{\pi l}{k}}\right\}_{l=0}^{K-1},\left\{r_{m}\right\}_{m=K}^{2 K-1}=\left\{-e^{j \frac{\pi l}{K}}\right\}_{l=0}^{K-1} .
$$

$\operatorname{Using}(15)$, we can get $\underline{y}_{l}(n)=\left[\mathrm{Y}\left(\mathrm{n} ; \mathrm{e}^{j \frac{\pi l}{K}}\right), \mathrm{Y}\left(n ;-\mathrm{e}^{j \frac{\pi l}{K}}\right)\right]^{T}$ as follows

$$
y_{l}(n)=\sqrt{K} \tilde{H}_{l} \underline{s}_{l}(n), \quad \forall l \in[0, K-1],
$$

where

$$
\tilde{H}_{l}=\left[\begin{array}{rr}
H\left(e^{j \frac{\pi l}{K}}\right) & e^{-j \frac{\pi l}{K}} H\left(e^{j \frac{\pi l}{K}}\right) \\
H\left(-e^{j \frac{\pi l}{K}}\right) & -e^{-j \frac{I}{K}} H\left(-e^{j \frac{\pi l}{K}}\right)
\end{array}\right]
$$

\section{SIMULATIONRESULTS}

In our simulation, the available channel bandwidth is assumed to be $1 M H z$. The entire bandwidth is divided into $\mathrm{K}=\mathbf{6 4}$ subchannels. To make the subcamers orthogonal to each other, the symbol duration on each subcamer, denoted by $T_{s}$, is $T_{s}=64 \mu s$. The duration of the IOFDM block is $128 \mu s$. A ZP of duration $T_{z}$ is used to avoid IBI due to the frequency selectivenature of the channel. The total duration of the transmitted IOFDM block is $128 \mu \mathrm{s}+\mathrm{T}$, where $T_{z}$ has been chosen according to channel model. The twelve path GSM TU and HT channel models (see Table 1) are used. We have chosen $T_{z}=7 \mu s$ in TU channel model and $T_{z}=20 \mu s$ in HT channel model. The direct form mutipath Rayleigh fading simulator [5] and QPSK modulation 


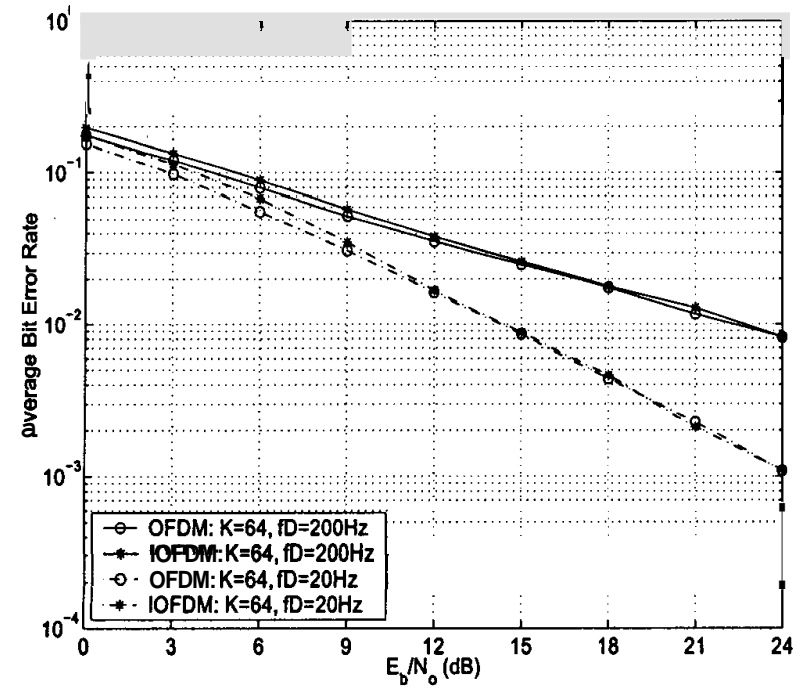

Fig. 3. Performance comparision of IOFDM system and OFDM system at different fading rates in TU channel model.

scheme are used. It is assumed that perfect CSI is available at the receiver.

The average Bit Error Rate (BER) is considered as the performance measure. To gain insights into the average BER performance of the OFDM system and the IOFDM system, we have taken 3000 OFDM blocks, of length $K=$ 64, per trial and averaged over 50 trials. In effect, for each $E_{b} / N_{o}$ value considered, the BER values are obtainedbased on $19.2 \times 10^{6}$ bits.

In Fig. 3, the average BER performance of the proposed IOFDM system is compared with the conventional OFDM system at fadingrates $f_{D}=20 \mathrm{~Hz}$ and $f_{D}=200 \mathrm{~Hz}$ in TU channel model. In Fig. 4, the average BER performance of the IOFDM system is compared with the OFDM system at fading rates $f_{D}=20 \mathrm{~Hz}$ and $f_{D}=200 \mathrm{~Hz}$ in HT channel model. In both cases, the performance of the IOFDM system is very close to the OFDM system.

\section{CONCLUSION}

A new OFDM system based on interleavingthe elements of two consecutiveOFDM blocks has been presented. Simulation results verify that the code rate of the proposed IOFDM system is higher than the conventional OFDM system for no loss in performance. Generalizing this idea by considering more than two OFDM blocks is under progress [6].

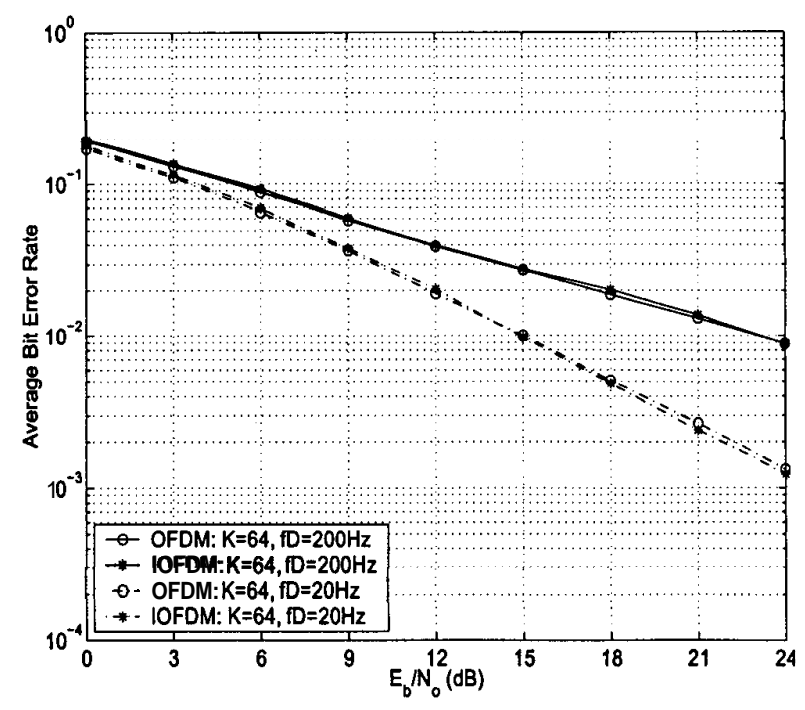

Fig. 4. Performance comparision of IOFDM system and OFDM system at different fading rates in HT channel model.

\section{REFERENCES}

[1] B.Muquet, M.de Courville, P.Duhamel and G.B.Giannakis, "OFDM with Trailing Zeros Versus OFDM with Cyclic Prefix: Links, Comparisions ans Application to the HIPERLAN/2 system," Proc. of ICC, New Orleans, LA, pp. 1049-1053, June 2000.

[2] Z.Liu, G.B.Giannakis, S.Barbarossa and A.Scaglione, "Transmit-antennae space-time block coding for generalized OFDM in the presence of unknown multipath," IEEE Journal on Selected Areas in Communications, vol. 19, no. 7, pp. 1352-1364,July 2001.

[3] L.J.Cimini, Jr., "Analysis and simulation of a digital mobile channel using Orthogonal Frequency Division Multiplexing," IEEE Transactions on Communications, vol. COM-33, no. 7, pp. 665-675, July 1985.

[4] J.A.C.Bingham, "Multicarrier modulation for data transmission: $\boldsymbol{A n}$ idea whose time has come," IEEE Communications Magazine, pp. 5-14, May 1990.

[5] S.A.Fechtel, "A novel approach to modeling and efficient simulation of frequency selective fading radio channels," IEEE Journal on Selected Areas in Communications, vol. 11, no. 3, pp. 422-431, April 1993.

[6] V.G.S.Prasad and K.V.S.Hari, "Generalized Interleaved Orthogonal Frequency Division Multiplexing system," underpreparation. 